\title{
My TechLife: Desenvolvimento e Validação de um Jogo para o Ensino Técnico em Informática
}

\author{
Diego Pinheiro da Silva, Universidade do Vale do Rio dos Sinos, \\ diegopinheiro@feevale.br \\ Marta Rosecler Bez, Universidade Feevale, martabez@gmail.com \\ Sandro José Rigo, Universidade do Vale do Rio dos Sinos, rigo@unisinos.br
}

Resumo. Este artigo apresenta a construção e a validação de um jogo RPG para o aprendizado de técnicas de informática, nomeado My TechLife. O Jogo simula a resolução de problemas reais vivenciados por técnicos em informática, consistindo em uma ferramenta educacional e motivadora para o aprendizado. Este foi desenvolvido na linguagem JavaScript, utilizando como ferramenta de apoio o Rpg Maker MV. O jogo está disponível para smartphones na plataforma Android. Ele foi validado por 52 alunos de cursos técnico de informática de 4 instituições de ensino, os quais jogaram e depois preencheram um questionário avaliativo. $\mathrm{Na}$ análise do questionário observase que o jogo foi muito bem avaliado como ferramenta a ser utilizada no ensino em informática.

Palavras-chave: Jogo educacional, Educação em Informática, Gamificação, Informática na Educação, My TechLife.

\section{My TechLife: Development and Validation of a Computer Education Game}

\begin{abstract}
This article presents the construction and validation of an game $R P G$ for the learning of computer techniques, named My TechLife. The Game simulates the resolution of real problems experienced by computer technicians, consisting of an educational and motivating tool for learning. This was developed in JavaScript language, using as support tool the Rpg Maker MV. The game is available for smartphones on the Android platform. It was evaluated by 52 students of computer technology courses from 4 educational institutions, who played and then filled out an evaluation questionnaire. In the analysis of the questionnaire it is observed that the game was very well evaluated as a tool to be used in teaching in computer science.
\end{abstract}

Keywords: Educational Game, Computer Education, Gamification, Computer Science in Education, My TechLife.

\section{Introdução}

Novas formas de recursos educacionais vêm sendo analisadas, dentre as quais destacamse os jogos. Através dos mesmos, pode-se ampliar o interesse no ensino e desta forma reduzir o abandono de adolescentes nas escolas, transformando a escola em um 
ambiente mais acolhedor e interessante para a nova geração (BALDEÓN et al., 2015). Os jogos são ferramentas capazes de potencializar aprendizagens em inúmeras áreas do conhecimento (AZEVEDO, 2012), (BOMFOCO, 2012).

Nos jogos educativos, os alunos são expostos a desafios, desenvolvem o raciocínio e estimula-se a tomada de decisão, mediante à resolução de problemas. $\mathrm{O}$ RPG (Rolling Player Game) é um gênero de jogo que possibilita simulações de qualquer realidade, mostrando-se eficaz como uma nova tecnologia de suporte à educação. Através dele, é possível fazer com que o estudante tenha interesse e motivação para buscar a informação necessária (PETRI \& CHIAVEGATTI, 2015). Junior et al. (2017) avaliaram uma estratégia de ensino usando o RPG PeaceMaker como entretenimento rico em conteúdo nas disciplinas de história e geografia, em três grupos de estudos. Para tanto, realizou um trabalho interdisciplinar oferecendo aos alunos uma maior abrangência e possibilidade de prender a atenção dos mesmos, melhorando sua aprendizagem e aprimorando o processo de ensino-aprendizagem.

No mesmo contexto deste artigo, apesar de não ser um jogo, Machado e Silva (2005) desenvolveram um objeto de aprendizagem para o ensino sobre a temática montagem de computadores para alunos de um curso técnico em informática. Foi desenvolvido em Flash, representando partes do computador que deveriam ser encaixadas. $\mathrm{O}$ aluno recebe uma folha para anotar as conexões e esta era entregue posteriormente ao professor. $\mathrm{O}$ objeto de aprendizagem foi validado com duas turmas, uma iniciante e outra avançada. Segundo os autores, na avaliação o jogo foi identificado como um meio eficiente a ser utilizado para o processo de ensino e aprendizagem da disciplina de manutenção de computadores.

Este artigo apresenta a construção e a validação de um jogo RPG para o ensino de técnicas de informática, especificamente manutenção e configuração de computadores, nomeado My TechLife. O jogo simula a resolução de problemas reais de técnicos de informática, tornando-se uma ferramenta educacional e motivadora para o aprendizado. Este foi desenvolvido na linguagem JavaScript, utilizando como ferramenta de apoio o Rpg Maker MV. Está disponível para smartphones na plataforma Android, tendo como público-alvo estudantes dos cursos de técnico de informática. $\mathrm{O}$ mesmo foi validado com 52 alunos do técnico de informática de quatro instituições de ensino particulares, os quais jogaram e após, preencheram um questionário.

Este artigo está dividido em cinco seções. Na segunda seção os materiais e métodos são descritos. Na seção três apresenta-se o desenvolvimento e a validação. A quarta seção apresenta a análise e discussões, seguida das conclusões.

\section{Material e Métodos}

Para o desenvolvimento do jogo foi usada a linguagem JavaScript, utilizando como ferramenta de apoio o Rpg Maker MV, software disponível pela Degica Company (disponível em rpgmakerweb.com/products/programs/rpg-maker-mv). Para jogos eletrônicos, o uso da linguagem de scripts é o mais indicado, evitando o processo de compilação e dando agilidade ao processo de criação (CRUZ \& ALBUQUERQUE, 2014). O jogo está disponível para smartphones na plataforma Android. A equipe de desenvolvimento foi composta por três professores, sendo dois universitários e um do ensino técnico em informática, com ampla vivência na temática do jogo. 
O My TechLife foi validado com 52 estudantes de Técnicos de Informática de 4 escolas particulares. Os alunos baixaram o jogo nos seus smartphones e, após a conclusão, preencheram um questionário avaliativo. Este foi elaborado com base em Bez (2013). O questionário é dividido em blocos de perguntas, buscando identificar a metodologia, as funcionalidades, a confiabilidade, a usabilidade, a eficiência e a aprendizagem proporcionada pelo jogo, com respostas baseadas na escala Likert com cinco opções, assim distribuídas: discordo totalmente, discordo, indiferente, concordo e concordo plenamente.

Durante a validação, foi analisado o comportamento dos alunos e as dúvidas anotadas para uma análise posterior. Os estudantes que participaram da validação possuem a idade entre 15 e 18 anos. Todos os respondentes indicaram ter conhecimento de informática e utilizam softwares como editores de textos, planilhas de cálculo, editores de apresentação e navegadores de internet.

\section{Desenvolvimento}

O jogo "My TechLife" é um RPG educacional, desenvolvido com o objetivo de gamificar o ensino nos cursos técnico em informática. Existe um alto grau de interesse de adolescentes em jogos digitais e, controverso a isso, muitas vezes, encontram-se desmotivados com o conteúdo tradicional do curso. Observa-se, também, o problema de que em determinadas atividades técnicas de informática, os computadores da escola são restritos e dificultam a aprendizagem. Um exemplo disso é a instalação do Windows, na maioria das vezes não permitida, recebendo, o aluno, apenas a teoria que abarca este conteúdo. Através do jogo, é possível simular esses cenários, de forma que os estudantes poderão aprender na prática e divertindo-se.

Esta primeira versão do jogo aborda as seguintes atividades: o objetivo geral do jogo é construir uma assistência técnica de informática, onde o jogador irá simular diversas situações e problemas reais, devendo buscar a solução para os mesmos. Inicialmente, o conteúdo é introduzido por um professor, que lhe pedirá informações como nome, gênero e aparência. Após o jogador construir seu personagem, um pequeno tutorial sobre os comandos do jogo é apresentado. A história tem seu início na formatura dos alunos do curso técnico de informática, do qual o protagonista estará se formando. No dia seguinte, o jogador começa com uma quantia de moeda fictícia e é estimulado a explorar o cenário virtual, onde deverá montar um ambiente de trabalho para sua assistência técnica, que vai desde escolher um estabelecimento, a comprar computadores e componentes de informática. Alguns exemplos de telas do jogo são ilustrados nas Figuras 1, 2, 3 e 4 .
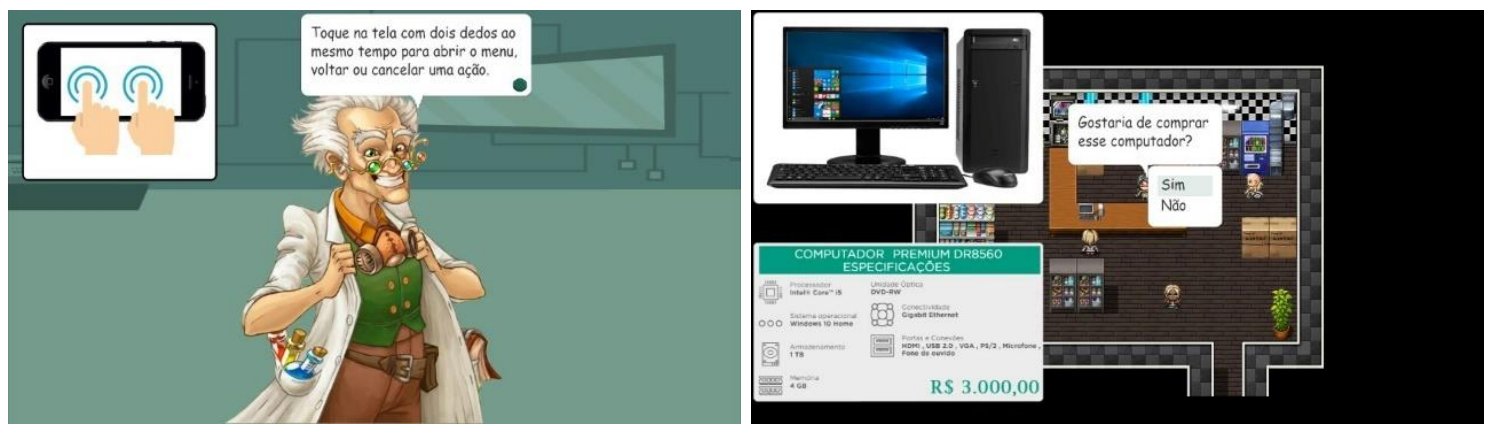

Figura 1. Tutorial de comandos (Do autor, 2018) Figura 2. Compra de um computador (Do autor, 2018) 


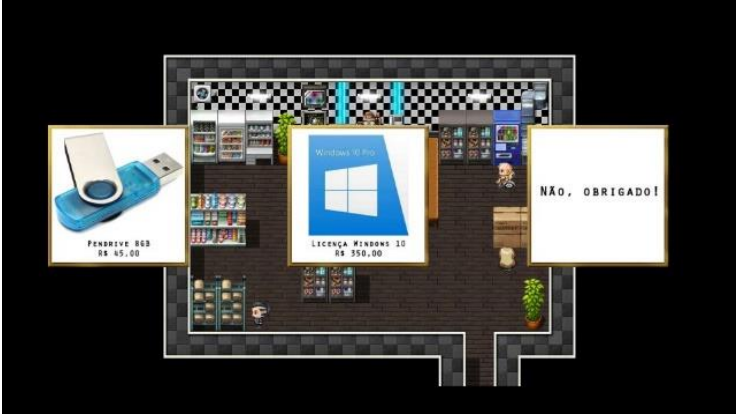

Figura 3. Compra de componentes (Do autor, 2018)

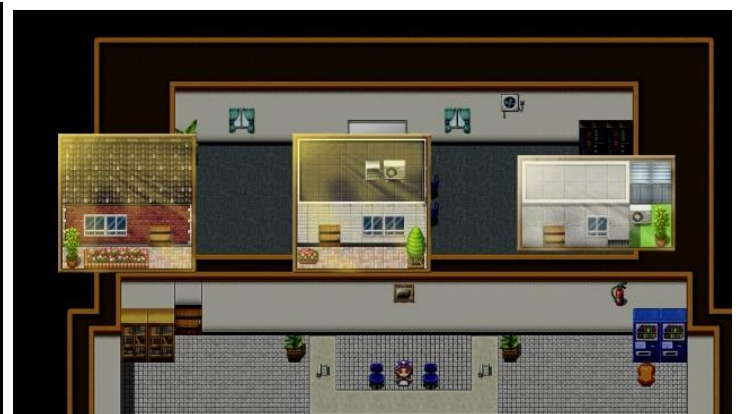

Figura 4. Compra de loja (Do autor, 2018)

$\mathrm{Na}$ área de trabalho, o protagonista receberá pedidos, como, por exemplo, instalar o Windows 10 , bem como a informação do computador recebido para reparo. Todo o processo de instalação é simulado com telas e opções reais, desde a criação de um Pendrive Bootável, a escolha de 32 ou 64 bits (conforme a capacidade do computador), a inserção de licença Microsoft (abordando temas como a pirataria), sendo que todos os passos serão considerados no feedback final do serviço. Caso o jogador não souber realizar a instalação ou ficar com dúvidas, o mesmo pode consultar nos livros presentes no cenário ou pedir instruções para seu pai (que na história, também é um técnico de informática). Alguns exemplos são ilustrados nas Figuras 5, 6, 7 e 8 .

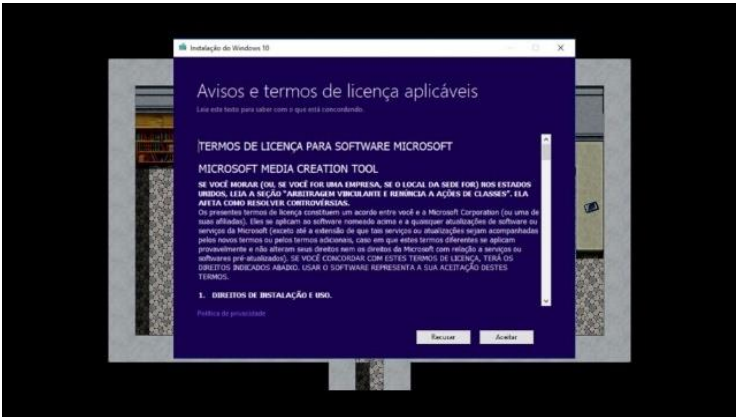

Figura 5. Criação de um boot (Do autor, 2018)

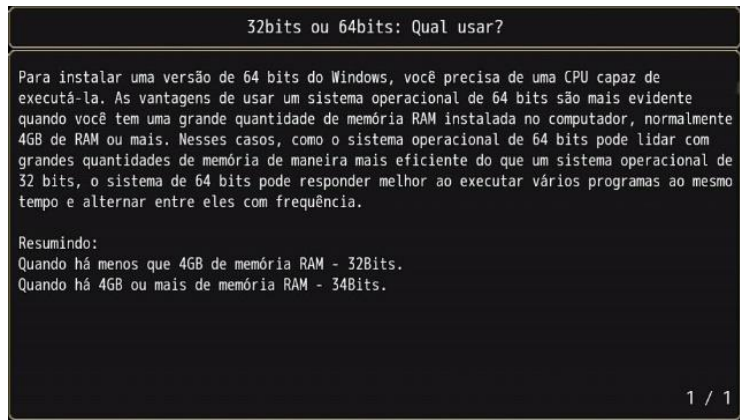

Figura 7. Livro disponível (Do autor, 2018)

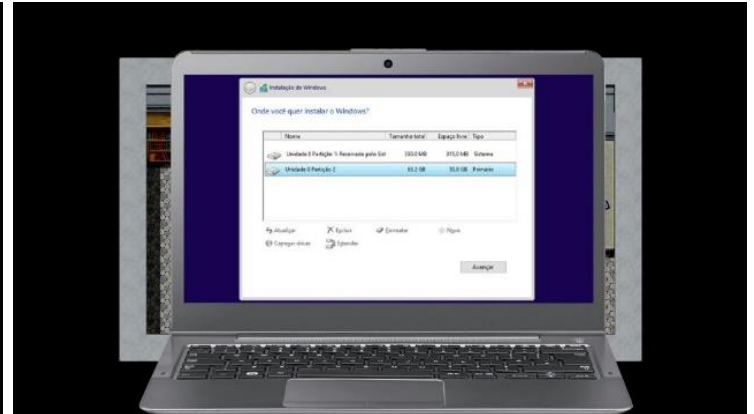

Figura 6. Unidade de Disco (Do autor, 2018)

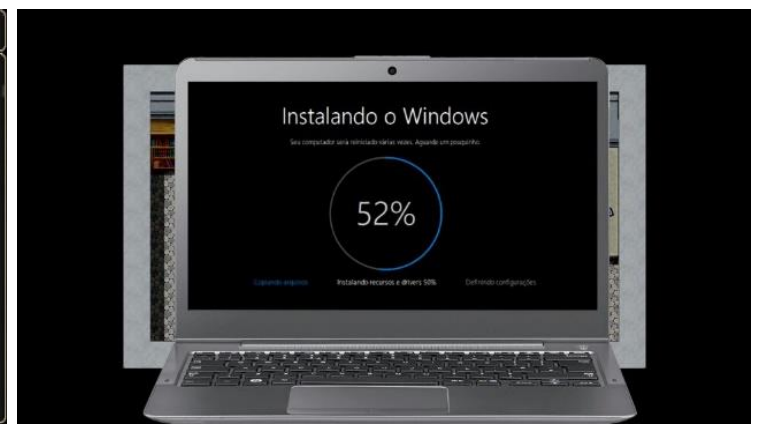

Figura 8. Instalação do Windows 10 (Do autor, 2018)

Após entregar o pedido (dar o mesmo como concluído), o jogador receberá o feedback de seu trabalho, com pontos de reputação. Os pontos recebidos podem ser positivos ou negativos, conforme os erros e acertos feitos pelo usuário. Como exemplo de ponto positivo, tem-se a instalação correta do tipo de bits do sistema. Um exemplo negativo seria entregar o computador com o Windows pirata. Um exemplo de Feedback Positivo é ilustrado nas Figuras 9 e 10. 


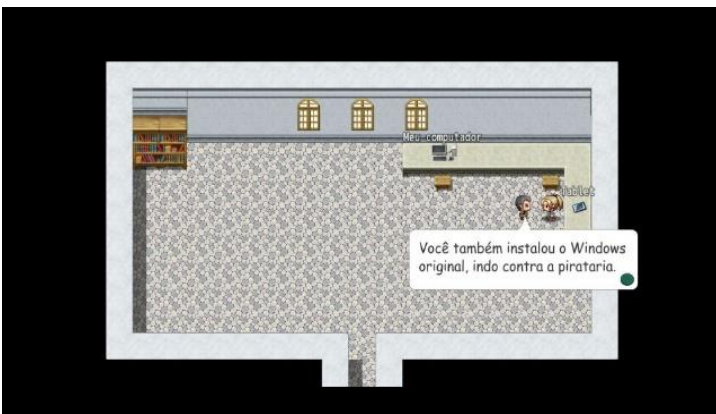

Figura 9. Feedback Positivo. (Do autor, 2018)

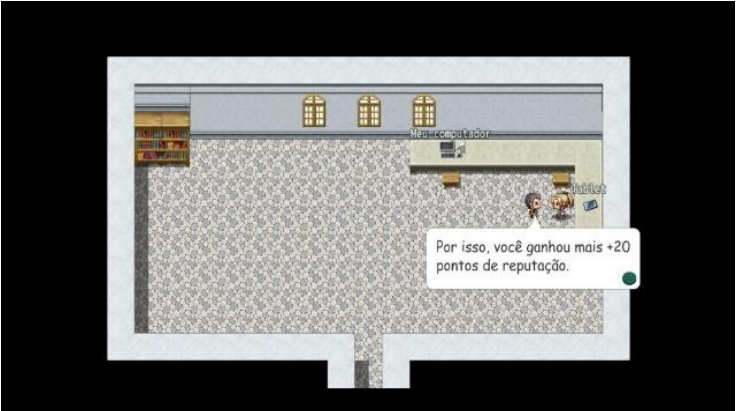

Figura 10. Distribuição de pontos. (Do autor, 2018)

Além do ambiente de trabalho do protagonista, o jogo apresenta uma cidade inteira, onde o jogador pode interagir com NPCs (Non-Player Character) e pode ter outros objetivos característicos, como jogar videogame, andar de carro, etc. O jogo desenvolvido tem como perspectiva a aprendizagem tangencial que está ancorada na facilitação dos processos de aprendizagem. A aprendizagem tangencial baseia-se na ideia de assimilar melhor as informações que interessa e que os jogos, de alguma forma, despertam o interesse por certos conteúdos (LEITE, 2016). Na Figura 11 é ilustrada a cidade do protagonista, e na Figura 12 um exemplo de mini game dentro do jogo.

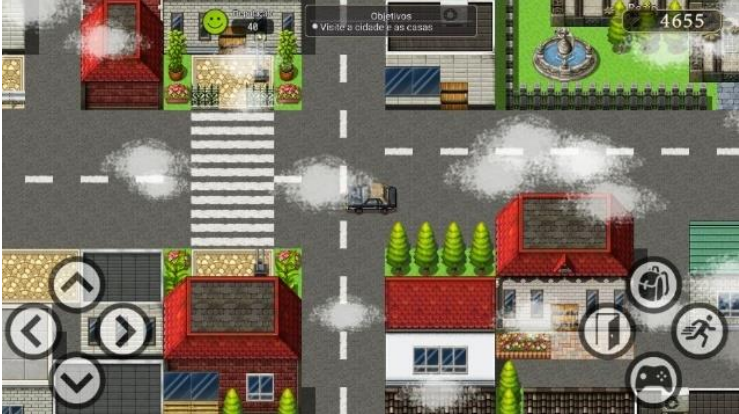

Figura 21. Cenário da cidade (Do autor, 2018)

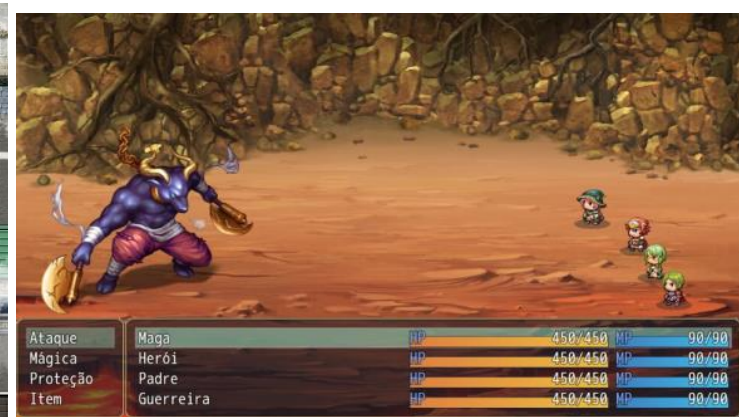

Figura 12. Exemplo de mini game (Do autor, 2018)

\section{Resultados e Discussão}

Na sequência, é apresentada uma análise a partir dos resultados obtidos. A comparação é realizada por grupos de perguntas, tal qual a divisão apresentada no questionário entregue aos participantes. Para cada bloco de perguntas, é apresentada uma análise detalhada dos resultados encontrados. A tabela 1 apresenta o resultado dos questionários aplicados.

Tabela 1. Respostas dos alunos (do autor, 2018)

\begin{tabular}{l|c|c|c|c|c}
\hline & $\begin{array}{l}\text { Concordo } \\
\text { plenamente }\end{array}$ & Concordo & Indiferente & Discordo & $\begin{array}{l}\text { Discordo } \\
\text { totalmente }\end{array}$ \\
\hline \multicolumn{4}{c}{ Metodologia utilizada no jogo } \\
$\begin{array}{l}\text { O game propicia uma fácil indicação de } \\
\text { instrução de técnicas de informática. }\end{array}$ & 39 & 12 & 1 & & \\
\hline $\begin{array}{l}\text { Os recursos disponibilizados são suficientes } \\
\text { para o estudo proposto, como informações de } \\
\text { texto e imagens. }\end{array}$ & 30 & 20 & 2 & & \\
\hline
\end{tabular}

Funcionalidades presentes no jogo

A descrição inicial e sequencial do jogo é clara e objetiva, levando o usuário a entender

50

2 


\begin{tabular}{l|c|c|c|c|c}
\hline & $\begin{array}{l}\text { Concordo } \\
\text { plenamente }\end{array}$ & Concordo & Indiferente & Discordo & $\begin{array}{l}\text { Discordo } \\
\text { totalmente }\end{array}$ \\
\hline o que deve ser realizado. & & & & \\
\hline $\begin{array}{l}\text { O aplicativo dispõe de funções que permitam } \\
\text { a adequada execução do jogo. }\end{array}$ & 47 & 4 & 1 & & \\
\hline $\begin{array}{l}\text { Percebo no jogo informações íntegras e } \\
\text { confiáveis. }\end{array}$ & 51 & 1 & & & \\
\hline $\begin{array}{l}\text { O jogo é preciso nos resultados parciais e } \\
\text { finais. }\end{array}$ & 42 & 7 & 2 & & \\
\hline $\begin{array}{l}\text { No jogo são atendidos os preceitos éticos e } \\
\text { morais. }\end{array}$ & 42 & 7 & 3 & & \\
\hline
\end{tabular}

Confiabilidade quanto ao uso do jogo

O jogo apresenta erros com frequência.

O jogo informa de forma clara quando ocorrem erros.

O jogo informa ao usuário a entrada de dados inválida.

O jogo é capaz de se recuperar em caso de falha.

\begin{tabular}{|l|l|l|l|l} 
& 1 & 2 & 3 & 46 \\
\hline 49 & 2 & & 1 & \\
\hline 46 & 4 & 2 & & \\
\hline 49 & 1 & 1 & 1 & \\
\hline
\end{tabular}

Usabilidade do jogo

\begin{tabular}{l|c|c|c|c|c}
\hline A interface do jogo facilita seu uso intuitivo. & 50 & 1 & 1 & & \\
\hline $\begin{array}{l}\text { As funções no jogo são fáceis de serem } \\
\text { executadas. }\end{array}$ & 52 & & & & \\
\hline O jogo é fácil de aprender a usar. & 47 & 3 & 2 & & \\
\hline $\begin{array}{l}\text { Existe clareza no conteúdo de ajuda } \\
\text { apresentado durante a execução do jogo. }\end{array}$ & 49 & 1 & 1 & 1 & \\
\hline
\end{tabular}

\section{Eficiência do Jogo}

$\begin{aligned} & \text { O tempo de resposta nas interações com o } \\ & \text { jogo é adequado. }\end{aligned}$
$\begin{aligned} & \text { O tempo de execução de cada caso é } \\ & \text { adequado. }\end{aligned}$

Aprendizagem no uso do Jogo

\begin{tabular}{l|c|c|c|c|c}
\hline $\begin{array}{l}\text { O jogo permite que o usuário adquira } \\
\text { conhecimento. }\end{array}$ & 51 & 1 & & & \\
\hline $\begin{array}{l}\text { O jogo é uma ferramenta motivacional para } \\
\text { aprendizagem. }\end{array}$ & 46 & 6 & & & \\
\hline O feedback do jogo ao aluno é adequado. & 40 & 10 & 2 & 1 & \\
\hline $\begin{array}{l}\text { O jogo permite uma maior participação do } \\
\text { aluno, interferindo na relação pedagógica } \\
\text { professor x aluno. }\end{array}$ & 49 & 3 & & & \\
\hline $\begin{array}{l}\text { O jogo favorece ao aluno a estudar de forma } \\
\text { autônoma. }\end{array}$ & 49 & 3 & & & \\
\hline $\begin{array}{l}\text { O jogo pode ser utilizado como um recurso } \\
\text { efetivo na educação }\end{array}$ & 51 & 1 & & & \\
\hline O jogo ensina questões técnicas de & 47 & 3 & 2 & & \\
\hline
\end{tabular}


Em termos de metodologia utilizada, o jogo foi muito bem avaliado. Quando questionados se o jogo propicia uma fácil indicação de instrução de técnicas de informática, $98 \%$ dos entrevistados concordaram e $2 \%$ dos participantes sentiram-se indiferente a esse fato. De forma semelhante, $96 \%$ dos participantes concordaram que os recursos disponibilizados são suficientes para o estudo proposto, como informações de texto e imagens, e 4\% dos participantes sentiram-se indiferentes a isso.

A partir desses resultados, verificou-se que, através do jogo, é possível implementar uma metodologia de estudos de técnicas de informática, e que os recursos disponibilizados aos usuários, em geral, foram suficientes para o aprendizado. Soares (2013) havia concluído, em seus estudos, que os métodos de ensino-aprendizagem estão se adaptando às questões da era digital, e o RPG possibilita uma vivência de conteúdo e experimentação, desenvolvendo habilidades de autonomia, cooperação e criatividade.

Em termos de funcionalidades, de forma geral, o jogo foi bem avaliado. Quando questionados se a descrição inicial e sequencial do jogo é clara e objetiva, levando o usuário a entender o que deve ser realizado, $100 \%$ dos respondentes concordaram. No quesito funções que permitam a adequada execução do jogo, 98\% dos respondentes concordaram, e $2 \%$ sentiram-se indiferentes.

Quanto aos quesitos integridade e confiabilidade das informações no jogo, 100\% dos respondentes indicaram a sua existência. Em termos de precisão nos resultados parciais e finais, $96 \%$ dos respondentes concordaram que o jogo apresenta resultados precisos, enquanto $4 \%$ mostraram-se indiferentes. Quanto ao atendimento dos preceitos éticos e morais, 94\% dos respondentes indicaram que esses são atendidos e 6\% mostraram-se indiferentes a esse fato.

É possível averiguar que as funcionalidades do jogo se apresentaram de forma fácil e clara, fazendo com que os usuários, desde o início, soubessem as funções permitidas dentro do jogo e possibilitando a execução do ensinamento proposto. Botezatu et al. (2010), em seu experimento, conclui que os quesitos de execução adequada são importantes para alcançar o objetivo educacional. A integridade e a confiabilidade evidenciam que registros reais ou mais próximos da realidade tornam mais efetivos os conteúdos estudados.

A confiabilidade no uso do jogo também apresentou um resultado satisfatório. Quando os participantes foram questionados se o jogo apresenta erros com frequência, $2 \%$ indicaram que sim, $5 \%$ foram indiferentes a esse aspecto e $93 \%$ discordaram. Foi questionado se, ao ocorrer um erro no jogo, esse é informado de forma clara. Neste quesito, $100 \%$ responderam que sim. Em termos de aviso sobre informações inválidas digitadas no jogo, 96\% dos respondentes indicaram que o sistema avisa em caso de digitação inválida e 4\% mostraram-se indiferentes à existência desses avisos. Sobre a possibilidade do jogo se recuperar em caso de falhas, $96 \%$ concordaram que isso ocorra, $2 \%$ responderam ser indiferentes ao fato e $2 \%$ discordaram.

Durante a execução, foi possível verificar que o jogo não apresentou nenhum problema, exceto por uma situação onde o dispositivo encerrou o mesmo sem recuperação. Após a análise da situação, verificou-se que o problema era causado pela gama de aplicativos abertos ao mesmo tempo, tornando a causa do problema $o$ 
dispositivo. Nielsen (1993) informa que, por melhor que seja a mensagem de falha exibida ao usuário, um bom software é aquele que impede a ocorrência dos problemas.

Em termos de usabilidade do jogo, de forma geral, foi bem avaliado. Quando os participantes foram questionados se a interface do jogo facilita o seu uso intuitivo, $98 \%$ dos participantes concordaram que o sistema é intuitivo e $2 \%$ mostraram-se indiferentes a este aspecto. No que se refere à facilidade de executar as funções no jogo, $100 \%$ indicaram ser fácil.

Quanto à facilidade de aprendizado no uso do jogo, apresentou-se um índice de $96 \%$ concordando e $2 \%$ mostraram-se indiferente ao fato. Questionou-se se há clareza no conteúdo de ajuda durante a execução do jogo. 96\% dos participantes responderam que sim, $2 \%$ mostraram-se indiferentes a este quesito e $2 \%$ discordaram.

Os resultados positivos indicam que a forma com que a interface do jogo foi programada se tornou eficaz, permitindo ao jogador utilizar tanto o modo touchscreen da tela como o modo joysticker, que são apresentados em um pequeno tutorial logo no início do jogo. Os comandos são fáceis e a indicação dos objetivos na tela facilitaram a usabilidade do usuário no game.

Em termos da eficiência do jogo, de forma geral, foi muito bem avaliado. Quando os participantes foram questionados se o tempo de resposta das interações é adequado, indicou-se $96 \%$ de concordância e $4 \%$ se sentem indiferente ao fato. No que se refere ao tempo de execução de cada caso (problema a ser resolvido), 94\% dos respondentes indicaram ser suficiente, e $6 \%$ mostraram-se indiferentes.

Por questões de adaptação ao tempo de aprendizado de cada aluno, optou-se por não delimitar um tempo para a execução das tarefas e problemas propostos, assim sendo, o aluno pode buscar ajuda quando precisa obter mais informações, preocupandose em realizar a atividade de forma correta do que com uma velocidade determinada.

Em termos de aprendizagem no uso do jogo, de forma geral, foi muito bem avaliado. Quando questionado se, na opinião dos participantes, o jogo permite que o usuário obtenha conhecimento, $100 \%$ dos respondentes indicaram que sim. Foi questionado aos participantes se o jogo pode ser considerado uma ferramenta motivacional para a aprendizagem, 100\% dos respondentes acreditam que sim.

Quando questionados se o feedback do jogo disponibilizado aos alunos é adequado, 94\% responderam ser adequado, $4 \%$ se mostraram indiferente ao fato e $2 \%$ indicaram que o feedback não é adequado. No que se refere a maior participação do aluno, interferindo na relação pedagógica professor $\mathrm{x}$ aluno, $100 \%$ dos respondentes indicaram que permite.

Quando questionados se o jogo favorece a autonomia do aluno, 100\% responderam positivamente. Da mesma forma, se o jogo pode ser utilizado como um recurso efetivo na educação de técnicas de informática, $100 \%$ dos respondentes indicaram que sim. Quando questionados se o jogo ensina questões técnicas de informática de maneira séria e divertida, 96\% concordaram e 4\% dos participantes se mostraram indiferente ao fato.

Através desse tópico, é possível verificar que o objetivo principal desta pesquisa foi atingido, onde se averiguou que é possível adquirir conhecimento através do jogo, e que a ferramenta é um método motivacional para a aprendizagem, resolvendo um dos principais problemas levantados. Pessini et al. (2015) ressaltam a importância da 
preparação dos estudantes para serem autônomos, capazes de cooperar, de lidar com as novas tecnologias e ter criatividade.

\section{Conclusões}

Esta pesquisa apresentou a construção e a validação de um game RPG para o ensino de técnicas de informática. O jogo simula a resolução de problemas reais vivenciados por técnicos de informática, tornando-se uma ferramenta educacional e motivadora para $o$ aprendizado. $\mathrm{O}$ mesmo foi validado com 52 alunos dos cursos técnico de informática de quatro instituições de ensino particulares, onde jogaram e preencheram um questionário avaliativo.

Os resultados da validação foram positivos, mostrando que foi possível adquirir conhecimento através do jogo e que a ferramenta é um método motivacional para a aprendizagem, resolvendo um dos principais problemas levantados. As funcionalidades do jogo apresentaram-se de forma fácil e clara, fazendo com que os usuários conseguissem realizar, desde o início, a execução do ensinamento proposto. A proposta do jogo como um método de aprendizagem alternativo atingiu os seus objetivos.

$\mathrm{O}$ diferencial do jogo aqui apresentado e validado, refere-se à possibilidade de aprendizado na prática de técnicas que não seriam possíveis na estrutura da escola, em função de bloqueios inerentes a segurança nos laboratórios de informática. Outro aspecto a destacar é a possibilidade de o aluno usar o aplicativo nos mais diversos locais, permitindo que este estude de forma autônoma e sem as barreiras geográficas e/ou temporais.

Como trabalhos futuros, serão inseridos diversos novos cenários e problemas de informática a serem resolvidos, bem como, expandir a área de estudo, como técnicas de programação, redes de computadores, entre outras. Será lançada uma versão para computadores e smartphones da plataforma iOS. Também serão realizados estudos para acrescentar novas funcionalidades que permitam facilitar e acrescentar materiais ao aprendizado dos estudantes, bem como, propostos os modelos de gamificação. O projeto será disponibilizado como open source para professores e pesquisadores, afim de inserir novos conteúdos e formas de aprendizagem.

\section{Referências}

AZEVEDO, V. A. Jogos eletrônicos e educação: construindo um roteiro para a sua análise pedagógica. Renote - Novas Tecnologias na Educação - UFRGS, Porto Alegre. V. 10, 2012.

BALDEÓN, J.; PUIG, A.; RODRÍGUEZ, I.; LOPEZ, M.; GRAU, S.; ESCAYOLA, M. Gamification of elementary math learning: A game Designer Role-Playing Experience With Kids. II International Workshop on Gamification in Education: gEducation 2015.

BEZ, M. R. Construção de um Modelo para o Uso de Simuladores na Implementação de Métodos Ativos de Aprendizagem das Escolas de Medicina. Porto Alegre, 2013. 314 f. Tese (Doutorado em Informática na Educação) - PGIE. CINTED - Universidade Federal do Rio Grande do Sul, POA.

BOMFOCO, M.A.; AZEVEDO, V.A. Os jogos eletrônicos e suas contribuições para a aprendizagem na visão de J. P. Gee. Renote - Novas Tecnologias na Educação UFRGS, Porto Alegre. V. 10 nº 3, 2012. 
BOTEZATU, M.; HULT, H.; TESSMA, M.; FORS, U. Virtual patient simulation: knowledge gain or knowledge loss?. Medical Teacher [serial online]. 2010;32(7):562-568. Available from: MEDLINE with Full Text, Ipswich, MA. Accessed June 27, 2017.

CRUZ, D. M.; ALBUQUERQUE, R. M. A produção de jogos eletrônicos por crianças: narrativas digitais e o RPG Maker / The production of electronic games by children: digital narratives and the RPG Maker. Revista Comunicação \& Educação, Ano XIX, número 1, jan/jun, 2014.

JUNIOR, S. A. F; PACHECO, M. G; JUNIOR, C. R. S.; BARRA, A. S. B. Metodologias de uso de jogo computacional RPG em sala de aula: estudo de caso com o jogo PeaceMaker. Revista Renote, V. 15 No 1, julho, 2017.

LEITE, B. S. Aprendizagem tangencial no processo de ensino e aprendizagem de conceitos científicos: um estudo de caso. Revista Renote, V. $14 \mathrm{~N}^{\mathrm{o}} 2$, dezembro, 2016 .

MACHADO, L. L.; SILVA, J. T. Objeto de aprendizagem digital para auxiliar o processo de ensino-aprendizagem no Ensino Técnico em Informática. Revista Renote, V.3 No 2, novembro, 2005.

NIELSEN, J. Usability Engineering. Boston - USA: Academic Press, 1993. 362 p.

PESSINI, A.; KENCZINSKI, A.; HOUNSELL, M. S. Uma Ferramenta de Autoria para o desenvolvimento de Jogos Sérios do Gênero RPG. Florianópolis: Computer on the Beach, 2015.

PETRI, G.; CHIAVEGATTI, N. C. Um Role Playing Game Para o Ensino de Elicitação e Análise de Requisitos. Revista Renote, V. 13 N 1, julho, 2015.

SOARES, A. N. Role Playing Game (Rpg): Elaboração e Avaliação de Estratégia Pedagógica Para Formação Crítica e Autônoma do Enfermeiro. Dissertação para conclusão de curso. Belo Horizonte, 2013. 